\title{
Thermodynamics and dielectric anomalies of DMAAS and DMAGaS crystals in the region of phase transitions (Landau theory approach)
}

\author{
I.V.Stasyuk ${ }^{1}$, O.V.Velychko ${ }^{1}$, Z.Czapla ${ }^{2}$, R.Czukwinski ${ }^{2}$ \\ 1 Institute for Condensed Matter Physics \\ of the National Academy of Sciences of Ukraine, \\ 1 Svientsitskii Str., 79011 Lviv, Ukraine \\ 2 Institute of Experimental Physics, University of Wroclaw, \\ 9 M.Borna Sq., 50-204 Wroclaw, Poland
}

Received December 22, 1999

\begin{abstract}
A simple description of thermodynamics of DMAAS and DMAGaS ferroelectric crystals by means of Landau expansion is proposed. Conditions of occurrence of phase transitions are established and their temperatures are obtained. The influence of external hydrostatic pressure on phase transitions is described. The temperature behaviour of dielectric susceptibility components and their anomalies in the vicinity of phase transition points are investigated. The results obtained are compared with the experimental data.
\end{abstract}

Key words: DMAAS, DMAGaS, ferroelectrics, phase transitions, Landau expansion

PACS: 77.84.-s, 64.60.Cn

\section{Introduction}

$\left(\mathrm{CH}_{3}\right)_{2} \mathrm{NH}_{2} \mathrm{Al}\left(\mathrm{SO}_{4}\right)_{2} \cdot 6 \mathrm{H}_{2} \mathrm{O}(\mathrm{DMAAS})$ and $\left(\mathrm{CH}_{3}\right)_{2} \mathrm{NH}_{2} \mathrm{Ga}\left(\mathrm{SO}_{4}\right)_{2} \cdot 6 \mathrm{H}_{2} \mathrm{O}(\mathrm{DMA}-$ $\mathrm{GaS})$ ferroelectric crystals are intensively studied in recent years. Their interesting feature is a possible existence of a crystal in the ferroelectric or in the antiferroelectric state depending on external conditions (e.g. temperature, hydrostatic pressure). There is a significant difference in the thermodynamical behaviour of crystals despite the isomorphism of their structure. At ambient pressure DMAGaS crystal has three phases: paraelectric $\left(T>T_{\mathrm{c}}\right)$, ferroelectric $\left(T_{1}<T<T_{\mathrm{c}}\right)$ and antiferroelectric $\left(T<T_{1}\right)$ with temperatures of phase transitions $T_{\mathrm{c}}=136 \mathrm{~K}$ (first order transition close to the tricritical point) and $T_{1}=117 \mathrm{~K}$ (first order transition). There are only two phases in DMAAS crystal at an ambient pressure: paraelectric $\left(T>T_{\mathrm{c}}\right)$ and ferroelectric $\left(T<T_{\mathrm{c}}\right)$ with $T_{\mathrm{c}}=155 \mathrm{~K}$. 
A set of structural [1-3], dilatometric, dielectric, pyroelectric and ultrasonic [412] measurements is made for considered systems, which allows us to establish their main dielectric, mechanical and dynamical characteristics (see below). At the same time these investigations are incomplete and of preliminary stage in many directions.

The nature of phase transitions in DMAAS and DMAGaS crystals was unclear up to the recent time. During the last years a conviction on the important role of dimethyl ammonium (DMA) groups in phase transitions due to their orientational ordering-disordering has been established (see, for example, [3,6,13-16]). In [17] the microscopic approach based on the order-disorder model accounting for different orientational states of DMA groups was proposed. In the framework of the model the phase transition to a ferroelectric state has been described and the conditions of realization of this transition as of the first or of the second order have been established. Order parameters of the system have been constructed. They are connected with the differences of occupancies of four possible positions of nitrogen ions corresponding to different orientations of groups. As a result of symmetry analysis it has been established that components of the order parameters belonging to irreducible representation $B_{u}$ of point symmetry group $2 / m$ of the high-temperature (paraelectric) phase describe ferroelectric ordering of DMA group along the ferroelectric axis OX (in crystallographic plane (ac)) as well as their antiferroelectric ordering along the OY axis (crystallographic axis b). The inverse ordering (the antiferroelectric along OX and the ferroelectric one along OY) corresponds to order parameter components belonging to the irreducible representation $A_{u}$. The appearing of nonzero order parameters of $B_{u}$ type turns the system into ferroelectric state (point group $m$ ) while nonzero order parameters of $A_{u}$ type cause an antiferroelectric state (point group 2).

Notwithstanding the further prospects of microscopic approach by means of the four-state order-disorder model, the more simple but more general thermodynamical description based on Landau expansion is of interest. One can construct corresponding Landau free energy and in a standard way investigate possible phase transitions and obtain criteria of their realization with the use of data of the above mentioned symmetry analysis. This is a main goal of the present work. The results obtained in the framework of Landau expansion will be used for interpreting the changes induced by the external pressure in the picture of phase transitions and for describing dielectric anomalies in the phase transition points of the crystals investigated.

\section{Thermodynamics of phase transitions (Landau theory approach)}

Let us describe the thermodynamics of phase transitions in DMAAS and DMAGaS crystals using the Landau expansion. We consider a simplified version when only one linear combination of the initial order parameter type is included for each of $B_{u}$ and $A_{u}$ irreducible representations. The combinations included are true order parameters: coefficients at their squared values tend to zero in the points of the corresponding second order transitions. 
Order parameters, which transform according to irreducible representations $B_{u}$ and $A_{u}$ of point symmetry group $2 / m$ of high-symmetry phase, are denoted as $\eta_{b}$ and $\eta_{a}$ correspondingly. The first parameter $\eta_{b}$ describes polarization of ferroelectric type along the OX axis with simultaneous antiferroelectric type ordering along the OY axis; the second one corresponds to the inverse orientation where antipolarization along OX is accompanied by polarization along OY.

We restrict ourselves to the case of the second order phase transition from the nonpolar high-temperature phase to the ordered one. In this case Landau expansion of free energy can be limited by terms of the fourth order:

$$
F=F_{0}+\frac{1}{2} a \eta_{a}^{2}+\frac{1}{2} b \eta_{b}^{2}+\frac{1}{4} c \eta_{a}^{4}+\frac{1}{4} d \eta_{b}^{4}+\frac{1}{2} f \eta_{a}^{2} \eta_{b}^{2}-E_{x} \eta_{b}-E_{y} \eta_{a}
$$

A linear dependence of coefficients $a$ and $b$ on temperature is assumed

$$
a=a^{\prime}\left(T-T_{\mathrm{c}}^{\prime}\right), \quad b=b^{\prime}\left(T-T_{\mathrm{c}}\right),
$$

where condition $T_{\mathrm{c}}>T_{\mathrm{c}}^{\prime}$ is satisfied for a normal state of the crystal which corresponds to the transition from the paraelectric phase to the ferroelectric phase as to the first one at lowering of the temperature.

Conditions of thermodynamical equilibrium correspond to the minimum of free energy and look like

$$
\begin{aligned}
& \frac{\partial F}{\partial \eta_{a}}=\eta_{a}\left(a+c \eta_{a}^{2}+f \eta_{b}^{2}\right)-E_{y}=0 \\
& \frac{\partial F}{\partial \eta_{b}}=\eta_{b}\left(b+d \eta_{b}^{2}+f \eta_{a}^{2}\right)-E_{x}=0 .
\end{aligned}
$$

At zero external fields there are following solutions

$$
\eta_{a}=\eta_{b}=0
$$

- paraphase (P-phase);

$$
\begin{aligned}
& \eta_{a}=0, \quad \eta_{b} \neq 0, \\
& \eta_{b 0}=\sqrt{-b / d}=\sqrt{b^{\prime}\left(T_{\mathrm{c}}-T\right) / d}
\end{aligned}
$$

- ferroelectric phase (F-phase);

$$
\begin{aligned}
& \eta_{a} \neq 0, \quad \eta_{b}=0, \\
& \eta_{a 0}=\sqrt{-a / c}=\sqrt{a^{\prime}\left(T_{\mathrm{c}}^{\prime}-T\right) / c}
\end{aligned}
$$

- antiferroelectric phase (AF-phase). ${ }^{1}$

Corresponding expressions for free energy in these phases are as follows

$$
\begin{aligned}
F_{(\mathrm{P})} & =F_{0}, \\
F_{(\mathrm{F})} & =F_{0}-\frac{1}{4 d} b^{2}\left(T-T_{\mathrm{c}}\right)^{2}, \\
F_{(\mathrm{AF})} & =F_{0}-\frac{1}{4 c} a^{\prime 2}\left(T-T_{\mathrm{c}}^{\prime}\right)^{2} .
\end{aligned}
$$

\footnotetext{
${ }^{1}$ We follow here the terminology widely used in literature on the subject.
} 
The phase transition $\mathrm{P} \rightarrow \mathrm{F}$ which is of the second order in the approximation used takes place at temperature $T_{\mathrm{c}}$. The phase transition $\mathrm{F} \rightarrow \mathrm{AF}$ which can take place at lower temperatures occurs at

$$
F_{(\mathrm{F})}=F_{(\mathrm{AF})} .
$$

The condition above determines the temperature of this first order phase transition:

$$
T_{1}=\frac{\varkappa T_{\mathrm{c}}^{\prime}-T_{\mathrm{c}}}{\varkappa-1}
$$

where

$$
\varkappa=\frac{a^{\prime} \sqrt{d}}{b^{\prime} \sqrt{c}}, \quad \varkappa>1 .
$$

Nonequalities

$$
0<T_{1}<T_{\mathrm{c}}
$$

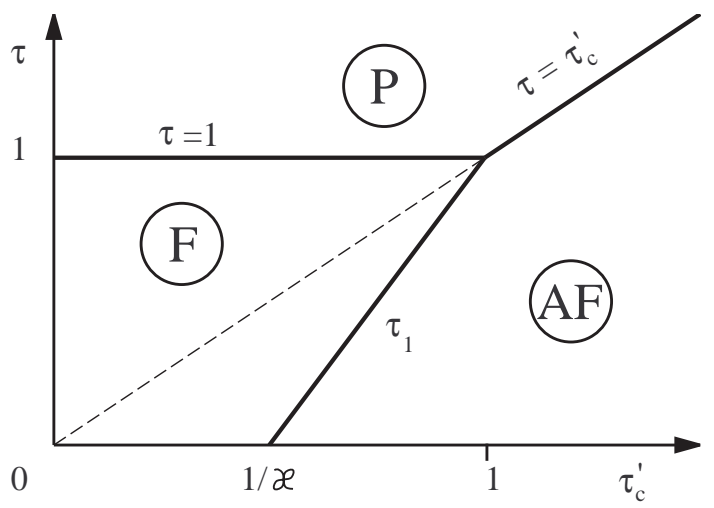

define the region of temperature $T_{\mathrm{c}}^{\prime}$ values where the F-phase exists as an intermediate one:

$$
\frac{1}{\varkappa}<\frac{T_{\mathrm{c}}^{\prime}}{T_{\mathrm{c}}}<1
$$

Figure 1. Dependence of phase number of the system and phase transition temperatures on values of system parameters $\left(\tau=T / T_{\mathrm{c}}, \tau_{\mathrm{c}}^{\prime}=T_{\mathrm{c}}^{\prime} / T_{\mathrm{c}}, \tau_{1}=\right.$ $\left.T_{1} / T_{\mathrm{c}}\right)$.

These conditions are illustrated by the phase diagram in figure 1 . In the case $T_{\mathrm{c}}^{\prime}>T_{\mathrm{c}}$ a direct phase transition $\mathrm{P} \rightarrow \mathrm{AF}$ from the paraelectric phase to the antiferroelectric one can take place.

The experimentally observed changes of temperatures of $\mathrm{P} \rightarrow \mathrm{F}$ and $\mathrm{F} \rightarrow \mathrm{AF}$ phase transitions and consecutive disappearance of the $\mathrm{F}$-phase as the result of increasing of the external hydrostatic pressure can be easily explained using the obtained diagram. Under the assumption that the effect of pressure leads mainly to shifts of temperatures $T_{\mathrm{c}}$ and $T_{\mathrm{c}}^{\prime}$

$$
\begin{aligned}
& T_{\mathrm{c}}=T_{\mathrm{c} 0}+x p, \\
& T_{\mathrm{c}}^{\prime}=T_{\mathrm{c} 0}^{\prime}+x^{\prime} p,
\end{aligned}
$$

and the changes of other Landau expansion parameters are negligible, the following relation is obtained

$$
T_{1}=T_{1}^{0}+\frac{\varkappa x^{\prime}-x}{\varkappa-1} p
$$

where

$$
T_{1}^{0}=\frac{\varkappa T_{\mathrm{c} 0}^{\prime}-T_{\mathrm{c} 0}}{\varkappa-1}
$$

According to the data published in [18], $\mathrm{d} T_{\mathrm{c}} / \mathrm{d} p \equiv x=-0.277 \mathrm{~K} / \mathrm{MPa} ; \partial T_{1} / \partial p=$ $1.95 \mathrm{~K} / \mathrm{MPa}$ and if one applies a linear approximation to the dependence of $T_{\mathrm{c}}^{\prime}$ on $p$ then $\mathrm{d} T_{\mathrm{c}}^{\prime} / \mathrm{d} p \equiv x^{\prime}=0.86 \mathrm{~K} / \mathrm{MPa}$. 
The obtained relations are illustrated by the diagram shown in figure 2. This diagram qualitatively matches the experimental (T,p) diagram for DMAGaS crystal (at $T_{\mathrm{c} 0}=136 \mathrm{~K}, T_{1}^{0}=116 \mathrm{~K}$ ) [18]. The obtained coordinates of triple point

$$
T_{3}=\frac{x^{\prime} T_{\mathrm{c} 0}-x T_{\mathrm{c} 0}^{\prime}}{x^{\prime}-x}, \quad p_{3}=\frac{T_{\mathrm{c} 0}-T_{\mathrm{c} 0}^{\prime}}{x^{\prime}-x},
$$

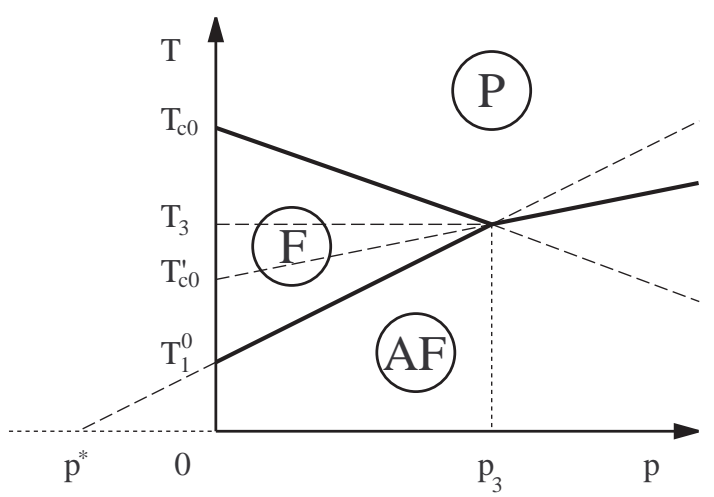

Figure 2. Dependence of the phase transition temperatures on the external hydrostatic pressure.

where lines of phase transitions $\mathrm{P} \rightarrow \mathrm{F}$, $\mathrm{F} \rightarrow \mathrm{AF}$ and $\mathrm{P} \rightarrow \mathrm{AF}$ come together are in good agreement with the experimental ones $\left(T_{3}^{\exp }=-140.3^{\circ} \mathrm{C} ; p_{3}^{\exp }=8.75 \mathrm{MPa}\right)$. At $p \gg p_{3}$ there is a deviation of the theoretical prediction of temperature of the $\mathrm{P} \rightarrow \mathrm{AF}$ phase transition from the experimental data. Unlike relationship (13) experimental dependence is nonlinear at large pressures.

The pressure value

$$
p^{*}=\frac{\varkappa T_{\mathrm{c} 0}^{\prime}-T_{\mathrm{c} 0}}{x-\varkappa x^{\prime}}
$$

(see figure 2) is an important characteristic of the model. At $p^{*}<0$, which is realized at $T_{\mathrm{c} 0}^{\prime} / T_{\mathrm{c} 0}>1 / \varkappa, \mathrm{AF}$-phase exists in the region of low temperatures at an ambient pressure (this situation takes place for DMAGaS). At $p^{*}>0$ (i.e. $T_{\mathrm{c} 0}^{\prime} / T_{\mathrm{c} 0}<1 / \varkappa$ ) and ambient pressure only P- and Fphases occur; this case can correspond to DMAAS crystal.

\section{Dielectric susceptibility}

The approach used in the previous section allows us to derive expressions for components of dielectric susceptibility tensor in the vicinity of phase transition points and to describe their temperature dependencies in general. In the approximation used, the components $P_{x}$ and $P_{y}$ of polarization vector are defined by parameters $\eta_{b}$ and $\eta_{a}$ correspondingly. Hence

$$
\chi_{x x}=\frac{\partial \eta_{b}}{\partial E_{x}}, \quad \chi_{y y}=\frac{\partial \eta_{a}}{\partial E_{y}}
$$

and proceeding from equations (3) one can obtain

$$
\chi_{x x}=\left(a+3 c \eta_{a}^{2}+f \eta_{b}^{2}\right) / D, \quad \chi_{y y}=\left(b+3 d \eta_{b}^{2}+f \eta_{a}^{2}\right) / D,
$$

where

$$
D=\left(a+3 c \eta_{a}^{2}+f \eta_{b}^{2}\right)\left(b+3 d \eta_{b}^{2}+f \eta_{a}^{2}\right)-4 f^{2} \eta_{a}^{2} \eta_{b}^{2} .
$$

The following particular cases follow from the expression (19): 
1. Paraphase $(\mathrm{P})$ :

$$
\chi_{x x}=\frac{1}{b}=\frac{1}{b^{\prime}\left(T-T_{\mathrm{c}}\right)}, \quad \chi_{y y}=\frac{1}{a}=\frac{1}{a^{\prime}\left(T-T_{\mathrm{c}}^{\prime}\right)} .
$$

2. Ferroelectric phase $(\mathrm{F})$ :

$$
\chi_{x x}=-\frac{1}{2 b}=\frac{1}{2 b^{\prime}\left(T_{\mathrm{c}}-T\right)}, \quad \chi_{y y}=\frac{1}{(\xi-1) a^{\prime}\left(T^{*}-T\right)},
$$

here the notations are used:

$$
T^{*}=T_{\mathrm{c}}+\frac{T_{\mathrm{c}}-T_{\mathrm{c}}^{\prime}}{\xi-1}, \quad \xi=\frac{f b^{\prime}}{d a^{\prime}} \quad(\xi>1) .
$$

In this case susceptibility $\chi_{y y}$ can be also expressed in the form

$$
\chi_{y y}=\left[a+f \eta_{b 0}^{2}\right]^{-1},
$$

where $\eta_{b 0}$ is a spontaneous value of the order parameter (polarization $P_{\mathrm{s}}$ ) in the ferroelectric phase.

3. Antiferroelectric phase $(\mathrm{AF})$ :

$$
\chi_{x x}=\frac{1}{\left(\varkappa^{2} \xi-1\right) b^{\prime}\left(T^{* *}-T\right)}, \quad \chi_{y y}=-\frac{1}{2 a}=\frac{1}{2 a^{\prime}\left(T_{\mathrm{c}}^{\prime}-T\right)},
$$

where the temperature

$$
T^{* *}=T_{\mathrm{c}}-\frac{T_{\mathrm{c}}-T_{\mathrm{c}}^{\prime}}{1-1 / \varkappa^{2} \xi},
$$

is introduced such that $T^{*}>T_{\mathrm{c}}>T^{* *}$. An expression similar to the previous one takes place

$$
\chi_{x x}=\left[b+f \eta_{a 0}^{2}\right]^{-1} .
$$

It relates the temperature dependence of longitudinal susceptibility in AF phase with the equilibrium value of the order parameter (polarization in one of sublattices).

The temperature behaviour of dielectric susceptibility components and their anomalies in the phase transition points are illustrated in figure 3 and 4 as temperature dependencies of inverse susceptibilities $\chi_{\alpha \alpha}^{-1}$.

The inverse susceptibility $\chi_{x x}^{-1}$ is equal to zero at the temperature $T_{\mathrm{c}}$. Its linear dependence on temperature in the vicinity of this point has an inclination $b^{\prime}$ at $T>T_{\mathrm{c}}$ and $2 b^{\prime}$ in the ferroelectric phase (figure 3 ). This typical behaviour for second order phase transition changes if the phase transition $\mathrm{P} \rightarrow \mathrm{F}$ is of the first order. Such a situation takes place in the DMAGaS crystal where the first order phase transition close to the tricritical point is observed. Then the susceptibility $\chi_{x x}^{-1}$ remains nonzero at $T_{\mathrm{c}}$ and has a small jump (according to data [11], $T_{\mathrm{c}}-T_{0} \simeq 1.2 \mathrm{~K}$, where $T_{0}$ is the 


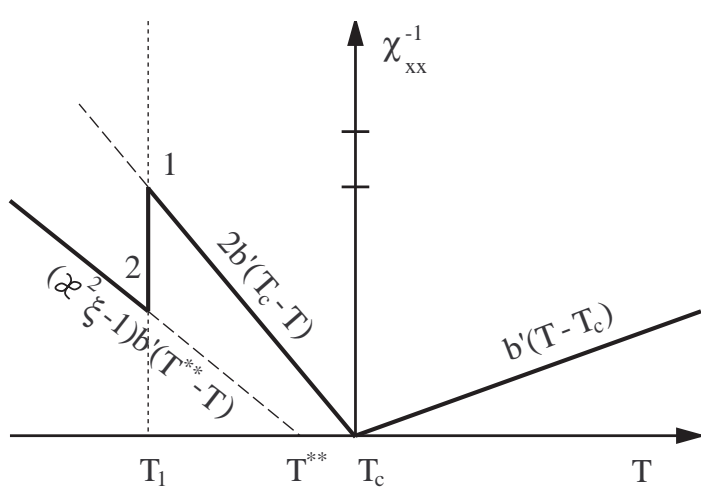

Figure 3. Temperature dependences of $\chi_{x x}^{-1}$ component of inverse susceptibility.

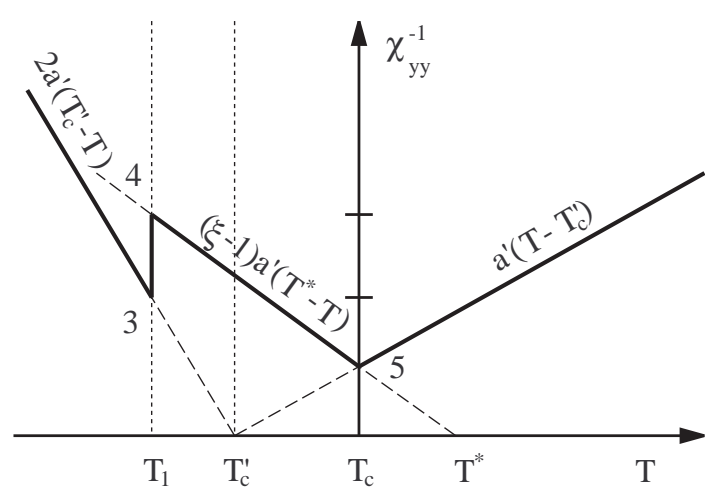

Figure 4. Temperature dependences of $\chi_{y y}^{-1}$ component of inverse susceptibility.

temperature at which $\left.\chi_{x x}^{-1} \rightarrow 0 ; \chi_{x x}^{-1}\left(T=T_{\mathrm{c}}\right) \simeq 6 \cdot 10^{-4}\right)$. The mentioned changes are relevant only to a small vicinity of $T_{\mathrm{c}}$. The phase transition $\mathrm{F} \rightarrow \mathrm{AF}$ is a well pronounced first order phase transition accompanied by jump of the $\chi_{x x}^{-1}$ function. The continuation of the straight line describing the temperature dependence of $\chi_{x x}^{-1}$ in the AF phase passes the point $T^{* *}$ (see figure 3). $\chi_{x x}^{-1}$ has the following values at the ends of its jump

$$
\left.\chi_{x x}^{-1}\right|_{1}=2 b^{\prime} \frac{\varkappa}{\varkappa-1}\left(T_{\mathrm{c}}-T_{\mathrm{c}}^{\prime}\right),\left.\quad \chi_{x x}^{-1}\right|_{2}=b^{\prime} \frac{\varkappa}{\varkappa-1}(\varkappa \xi-1)\left(T_{\mathrm{c}}-T_{\mathrm{c}}^{\prime}\right) .
$$

The value of susceptibility jump $\Delta \chi_{x x}^{-1}=\left.\chi_{x x}^{-1}\right|_{2}-\left.\chi_{x x}^{-1}\right|_{1}$ can be positive or negative depending on the values of theory parameters.

Temperature behaviour of the inverse susceptibility $\chi_{y y}^{-1}$ is essentially different. In the point of the second order phase transition $\mathrm{P} \rightarrow \mathrm{F}$ it remains nonzero with the value

$$
\chi_{y y}^{-1}\left(T_{\mathrm{c}}\right)=a^{\prime}\left(T_{\mathrm{c}}-T_{\mathrm{c}}^{\prime}\right)
$$

Its continuation to lower temperatures goes to zero at $T \rightarrow T_{\mathrm{c}}^{\prime}$. The continuation of the line of the inverse susceptibility in the antiferroelectric phase $\chi_{y y}^{-1}(T)=2 a^{\prime}\left(T_{\mathrm{c}}^{\prime}-\right.$ $T$ ) also goes across this point. In the ferroelectric phase region the function $\chi_{y y}^{-1}(T)$ is linear with the continuation passing the point $T^{*}$. At the $\mathrm{F} \rightarrow \mathrm{AF}$ phase transition this function has a jump between points

$$
\left.\chi_{y y}^{-1}\right|_{3}=\frac{2 a^{\prime}}{\varkappa-1}\left(T_{\mathrm{c}}-T_{\mathrm{c}}^{\prime}\right),\left.\quad \chi_{y y}^{-1}\right|_{4}=\frac{\varkappa \xi-1}{\varkappa-1} a^{\prime}\left(T_{\mathrm{c}}-T_{\mathrm{c}}^{\prime}\right) .
$$

Similar to the case of the function $\chi_{x x}^{-1}$ the jump can have positive or negative value.

\section{Discussion}

Proceeding from the formulae obtained in the previous section one can try to interpret the available data on the temperature dependence of dielectric susceptibility components of DMAAS and DMAGaS crystals. The majority of the performed 
measurements is devoted to the longitudinal dielectric permittivity $\varepsilon_{x}$ (or its real part $\varepsilon_{x}^{\prime}$ for low frequency alternating current measurements) mainly in the region of the high-temperature phase transition for DMAGaS and the corresponding phase transition in DMAAS. Such data are reported in the works $[5,8,9,11]$ (DMAGaS) and $[4,7,8]$ (DMAAS); only in the paper [7] the temperature behaviour of all permittivity components $\left(\varepsilon_{a}^{\prime}, \varepsilon_{b}^{\prime}, \varepsilon_{\mathrm{c}}^{\prime}\right)$ for DMAAS crystal in the wide range of temperatures (from $\simeq 90 \mathrm{~K}$ to $\simeq 280 \mathrm{~K}$ ) was measured. In some papers the dependence of spontaneous polarization on temperature in the ferroelectric phase was investigated and coercivity fields were measured $[8,9]$ (the value of $P_{\mathrm{s}}$ in the state close to saturation is about $1.4-1.9 \mathrm{C} / \mathrm{m}^{2}$ for DMAAS and $0.9-2.0 \mathrm{C} / \mathrm{m}^{2}$ for DMAGaS). A particular investigation of the $T_{\mathrm{c}}$ point vicinity in DMAGaS devoted to the influence of the external electric field on the first order phase transition point and the difference $T_{\mathrm{c}}-T_{0}$ is made in [11]. Based on the available experimental data, Curie-Weiss constant (from the paraphase side) is estimated as 2700-3060 K for DMAGaS crystal and 2700-3000 K for DMAAS crystal. The phase transition to the ferroelectric phase in DMAGaS crystal is of the first order and close to the tricritical point; this fact however does not affect the behaviour of $\chi_{x x}$ and $\chi_{y y}$ far from the $T_{\mathrm{c}}$ point.

The mentioned experimental data are incomplete, hence only partial comparison with the results of thermodynamical description is possible. For example one can obtain the values of the temperature $T_{\mathrm{c}}^{\prime}$, parameters $b^{\prime}$ and $\varkappa$ for the DMAGaS crystal $T_{\mathrm{c}}^{\prime}=125 \mathrm{~K}, \varkappa=2.22, b^{\prime}=0.33 \cdot 10^{-3} \mathrm{~K}^{-1}$ using the above mentioned data regarding the influence of the external hydrostatic pressure on phase transitions in DMAGaS crystal [18] and on the results of measurements of dielectric characteristics.

More comprehensive and selfconsistent evaluation of temperatures $T_{\mathrm{c}}^{\prime}, T^{*}$ and $T^{* *}$ as well as Landau expansion parameters (or parameters $a^{\prime}, b^{\prime}, \varkappa, \xi$ and $f$ ) by means of the relationships presented in this section become possible after goal-oriented investigations of temperature dependencies of $\chi_{x x}^{-1}$ and $\chi_{y y}^{-1}$ in a wide temperature interval including the regions of existence of all phases for DMAGaS and DMAAS. Proceeding from the obtained results it will be possible to ascertain suitability of the simple thermodynamical description where Landau expansion is limited to only one order parameter for each of $B_{u}$ and $A_{u}$ representations. Such a description is obviously much simplified comparing to the results of the microscopic approach based on the four-state model of order-disorder type [17]. The investigation of DMA group ordering in the configurational space of four orientational states needs twocomponent order parameters $\eta_{b}^{\alpha}\left(B_{u}\right)$ and $\eta_{a}^{\alpha}\left(A_{u}\right), \alpha=1,2$. This fact could complicate temperature dependencies of dielectric characteristics of the model even for a thermodynamical description in the framework of Landau expansion.

Furthermore, the expression for Landau expansion of free energy (1) considered here includes the terms up to the fourth order. A consistent description of the first order phase transition $\mathrm{P} \rightarrow \mathrm{F}$ and of the related dielectric anomalies demands the inclusion of the sixth order terms into the expansion. Such a generalization is necessary for a comprehensive description of experimental data and can be performed relatively easily. 


\section{References}

1. Pietraszko A., Łukaszewicz K., Kirpicznikowa L.F. // Polish J. Chem., 1993, vol. 67, p. $1877-1884$.

2. Pietraszko A., Łukaszewicz K. // Polish J. Chem., 1994, vol. 68, p. 1239-1243.

3. Pietraszko A., Łukaszewicz K., Kirpicznikowa L.F. // Polish J. Chem., 1995, vol. 69, p. 922-930.

4. Cach R., Dacko S., Czapla Z. // Phys. Stat. Sol. (b), 1989, vol. 116, p. 827-831.

5. Andreev E.F., Varikash V.M., Shuvalov L.A. // Izv. AN SSSR, ser. fiz., 1999, vol. 53, p. 572-574 (in Russian).

6. Sobiestinskas P., Grigas Y., Andreev E.F., Varikash E.M. // Phase Transitions, 1992, vol. 40 , p. 85 .

7. Kapustianik V., Bublyk M., Polovinko I., Sveleba S., Trybula Z., Andreev E. // Phase Transitions, 1994, vol. 49, p. 231-235.

8. Dacko S., Czapla Z. // Ferroelectrics, 1996, vol. 185, p. 143-146.

9. Pykacz H., Czapla Z. // Ferroelectrics Letters, 1997, vol. 22, p. 107-112.

10. Furtak J., Czapla Z. // Ferroelectrics Letters, 1997, vol. 23, p. 63-67.

11. Tchukvinskyj R., Cach R., Czapla Z. // Z. Naturforsch., 1998, vol. 53a, p. 105-111.

12. Czapla Z., Tchukvinskyj R. // Acta Phys. Polonica A, 1998, vol. 93, p. 527-530.

13. Kazimirov V.Yu., Rieder E.E., Sarin V.A., Belushkin A.V., Shuvalov L.A., Fykin L.E., Ritter C. // J. Korean Phys. Soc., 1998, vol. 32, p. S91-S93.

14. Dolinšek J., Klanjšek M., Arčon D., Hae Jin Kim, Selinger J., Žagar V. // Phys. Rev. B, 1999, vol. 59, p. 3460-3467.

15. Alsabbagh N., Michel D., Furtak J., Czapla Z. // Phys. Stat. Sol. (a), 1998, vol. 167, p. $77-87$.

16. Voelkel G., Alsabbagh N., Botcher R., Michel D., Czapla Z. // Phys. Stat. Sol. (b), 1999, vol. 215, No. 2, p. R5-R6.

17. Stasyuk I.V., Velychko O.V. // Journ. Phys. Studies, 2000 (in print).

18. Yasuda N., Kaneda A., Czapla Z. // Ferroelectrics, 1999, vol. 223, p. 71. 


\title{
Термодинаміка та діелектричні аномалії кристалів DMAGaS i DMAAIS в області фазових переходів (наближення теорії Ландау)
}

\author{
І.В.Стасюк ${ }^{1}$, О.В.Величко ${ }^{1}$, З.Чапля ${ }^{2}$, Р.Чуквінсський ${ }^{2}$ \\ 1 Інститут фізики конденсованих систем НАН України, \\ 79011 Львів, вул. Свєнціцького, 1 \\ 2 Інститут експериментальної фізики, Університет м. Вроцлав, \\ Польща, 50-204 Вроцлав, пл. М.Борна, 9
}

Отримано 22 грудня 1999 p.

Запропоновано простий опис термодинаміки сегнетоелектричних кристалів типу DMAAS і DMAGaS за допомогою розкладу Ландау. Встановлено умови реалізації фазових переходів і отримано їх температури. Описано зміни температур фазових переходів під впливом зовнішнього всестороннього тиску. Досліджено температурну поведінку компонент діелектричної сприйнятливості та їх аномалії в околі точок фазових переходів. Отримані результати порівнюються 3 експериментальними даними.

Ключові слова: DMAAIS, DMAGaS, сегнетоелектрики, фазові переходи, розклад Ландау

PACS: $77.84 .-s, 64.60 . \mathrm{Cn}$ 